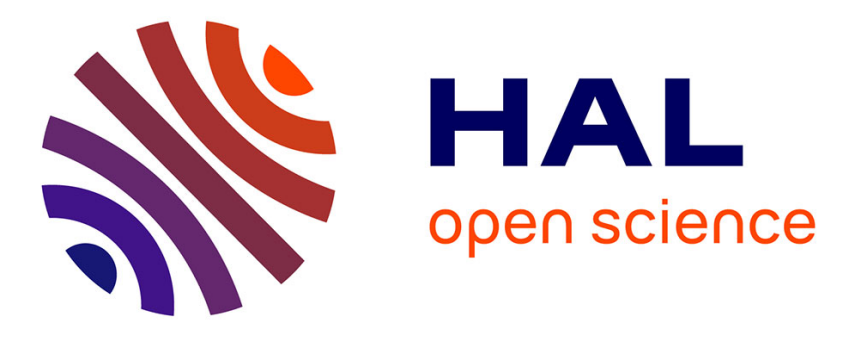

\title{
The Largest Connected Subgraph Game
}

Julien Bensmail, Foivos Fioravantes, Fionn Mc Inerney, Nicolas Nisse

\section{To cite this version:}

Julien Bensmail, Foivos Fioravantes, Fionn Mc Inerney, Nicolas Nisse. The Largest Connected Subgraph Game. WG 2021 - The 47th International Workshop on Graph-Theoretic Concepts in Computer Science, Jun 2021, Warsaw, Poland. pp.296-307, 10.1007/978-3-030-86838-3_23 . hal-03219636

\section{HAL Id: hal-03219636 \\ https://hal.inria.fr/hal-03219636}

Submitted on 6 May 2021

HAL is a multi-disciplinary open access archive for the deposit and dissemination of scientific research documents, whether they are published or not. The documents may come from teaching and research institutions in France or abroad, or from public or private research centers.
L'archive ouverte pluridisciplinaire HAL, est destinée au dépôt et à la diffusion de documents scientifiques de niveau recherche, publiés ou non, émanant des établissements d'enseignement et de recherche français ou étrangers, des laboratoires publics ou privés. 


\title{
The Largest Connected Subgraph Game ${ }^{\star}$
}

\author{
Julien Bensmail $^{1}$, Foivos Fioravantes ${ }^{1}$, Fionn Mc Inerney ${ }^{2}$, and Nicolas Nisse ${ }^{1}$ \\ 1 Université Côte d'Azur, CNRS, Inria, I3S, France \\ ${ }^{2}$ CISPA Helmholtz Center for Information Security, Saarbrücken, Germany
}

\begin{abstract}
We introduce the largest connected subgraph game played on an undirected graph $G$. In each round, Alice colours an uncoloured vertex of $G$ red, and then, Bob colours an uncoloured vertex blue, with no vertices initially coloured. Once all the vertices are coloured, Alice (Bob, resp.) wins if there is a red (blue, resp.) connected subgraph of order greater than the order of any blue (red, resp.) connected subgraph. If neither player wins, it is a draw. We first prove that Bob can never win, and define a large class of graphs (reflection graphs) in which the game is a draw. We show that determining the outcome of the game is PSPACEcomplete in bipartite graphs of small diameter, and that recognising reflection graphs is GI-hard. We prove that, the game is a draw in paths if and only if the path has even order or at least 11 vertices, and Alice wins in cycles if and only if the cycle is of odd order. We also give an algorithm computing the outcome of the game in cographs in linear time.
\end{abstract}

Keywords: Games on graphs, Scoring games, Connection games, PSPACE-complete.

\section{Introduction}

Games where players strive to make connected structures are connection games. Several of these games are well-known, like the game of Hex, introduced by Hein in 1942, and independently by Nash in 1948 [9]. Hex is played by two players on a hexagon-tiled board with two of its opposing sides coloured red and the other two blue. Each round, the first player colours an uncoloured tile red, and then, the second player colours one blue. The player that connects the two sides with his color wins. Another famous connection game is the Shannon switching game, invented by Shannon in the 1950s [10]. In this game, the first player wants to connect two marked vertices in a graph, and the second player wants to prevent this. The players take turns selecting edges of the graph, and the first player wins if there is a path consisting of only his edges between the two marked vertices. A variant where the players select vertices (and obtain their incident edges) also

* This work has been supported by the European Research Council (ERC) consolidator grant No. 725978 SYSTEMATICGRAPH, the STIC-AmSud project GALOP, the PHC Xu Guangqi project DESPROGES, and the UCA ${ }^{\mathrm{JEDI}}$ Investments in the Future project managed by the National Research Agency (ANR-15-IDEX-01). See [2] for the full version of the paper. 
exists. However, not all connection games involve connecting sides of a board or two vertices in a graph. For example, in Havannah, a board game invented by Freeling in 1981, the players may also win by forming closed loops, with the board and the rules similar to Hex. Connection games tend to be difficult complexitywise, which is a main reason they are played and studied. For example, Reisch proved that generalised Hex is PSPACE-complete [14], Even and Tarjan proved that the Shannon switching game on vertices (players select vertices, not edges) is PSPACE-complete [8], and Bonnet et al. proved that (generalised) Havannah is PSPACE-complete [3]. That being said, the Shannon switching game on edges is polynomial-time solvable [5]. For more on connection games, see [3,4].

Games in which the player with the largest score wins, are scoring games. The score in these games is an abstract quantity usually measured in a unit called points. Players may gain points in a myriad of ways, all depending on the rules of the game. For example, in the orthogonal colouring game [1], each player gets one point for each coloured vertex in their copy of the graph, and a player's final score is their total number of points. Recently, the papers [11,12] started to build a general theory around scoring games, and there have been many papers on different scoring games, such as $[7,13,16]$. In this paper, we introduce the following 2-player game that links connection and scoring games on graphs. For any graph $G$, the largest connected subgraph game is played between the first player, Alice, and the second player, Bob. Initially, no vertices are coloured. In each round, Alice first colours an uncoloured vertex of $G$ red, and then, Bob colours an uncoloured vertex blue. Each vertex can only be coloured once and its

colour cannot be modified. The game ends when every vertex in $G$ is coloured. If there is a connected red (blue, resp.) subgraph whose order (number of vertices) is strictly greater than the order of any connected blue (red, resp.) subgraph, then Alice (Bob, resp.) wins. If the order of the largest connected red subgraph equals the order of the largest connected blue subgraph, then the game is a draw.

We first define notations and prove preliminary results for the largest connected subgraph game in Sec. 2, i.e., showing that Bob never wins, that the game is a draw in a large class of graphs we call reflection graphs, and that recognising reflection graphs is GI-hard. In Sec. 3, we prove that the game is PSPACE-complete in bipartite graphs of diameter 5 . We then study the game in particular graph classes, with the resolution of the game for paths and cycles in Sec. 4, and a linear-time algorithm for solving the game in cographs in Sec. 5. These graph classes interestingly illustrate different types of playing strategies that Alice and Bob can employ. Lastly, we finish with open questions in Sec. 6.

\section{$2 \quad$ Notations and First Results}

In this section, we define notations and give preliminary results for the game. For any graph $G$, if Alice (Bob, resp.) has a winning strategy in the largest connected subgraph game, then $G$ is $A$-win ( $B$-win, resp.). If neither Alice nor Bob has a winning strategy in the largest connected subgraph game, i.e., it is a 
draw if both players use optimal strategies, then $G$ is $A B$-draw. Since it is never a disadvantage to play an extra turn, by the classic strategy stealing argument:

Theorem 1. [2] There does not exist a graph $G$ that is B-win.

Since there are no $B$-win graphs, the next natural question to ask is whether there are $A$-win ( $A B$-draw, resp.) graphs. There are an infinite number of $A$-win graphs as any star is $A$-win (Alice first colours the universal vertex). This also shows that there are an infinite number of graphs for which the order of the largest connected red subgraph is arbitrarily bigger than that of the blue one. There are also an infinite number of $A B$-draw graphs, since any graph of even order with two universal vertices is $A B$-draw. In Section 4, we show that any path of order at least 10 is $A B$-draw, and hence, that there are an infinite number of graphs of odd order that are $A B$-draw. We can actually define a much richer class of $A B$-draw graphs. A reflection graph is any graph $G$, whose vertices can be partitioned into two sets $U=\left\{u_{1}, \ldots, u_{n}\right\}$ and $V=\left\{v_{1}, \ldots, v_{n}\right\}$ such that:

1. there is an isomorphism between the subgraph induced by $U$ and the subgraph induced by $V$, where $v_{i}$ is the image of $u_{i}$, for all $1 \leq i \leq n$;

2. for any two vertices $u_{i} \in U$ and $v_{j} \in V$, if $u_{i} v_{j} \in E(G)$, then $u_{j} v_{i} \in E(G)$.

Theorem 2. [2] Any reflection graph $G$ is AB-draw.

Indeed, a drawing strategy for Bob is to colour $v_{i}\left(u_{i}\right.$, resp.), whenever Alice colours $u_{i}\left(v_{i}\right.$, resp.). Any even-order graph that is a path, cycle, or Cartesian product of two graphs, is a reflection graph. We prove that recognising reflection graphs is not in $\mathrm{P}$ unless the Graph Isomorphism problem is:

Theorem 3. [2] Given a graph $G$, deciding if $G$ is a reflection graph is GI-hard.

\section{Complexity}

In this section, we show that the largest connected subgraph game is PSPACEcomplete, even in bipartite graphs of small diameter. Our reduction is via POS $\mathrm{CNF}$, which was shown to be PSPACE-complete in [15], and is as follows:

POS CNF: 2-player game whose input is a set of variables $X=\left\{x_{1}, \ldots, x_{n}\right\}$ and a conjunctive normal form (CNF) formula $\phi$ consisting of clauses $C_{1}, \ldots, C_{m}$ comprised of variables from $X$ in their positive form. In each round, the first player, Alice, sets a variable (that is not yet set) to true, and then, the second player, Bob, sets a variable (that is not yet set) to false. Once each variable has been assigned a truth value, Alice wins if $\phi$ is true, and Bob wins if $\phi$ is false.

Theorem 4. Given a graph $G$, deciding if $G$ is $A$-win is PSPACE-complete, even if $G$ is bipartite and has a diameter of 5 . 


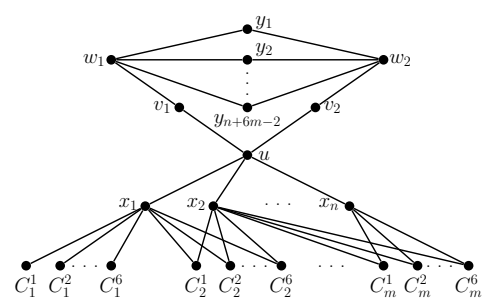

Fig. 1. An example of the construction of the graph $G$ in the proof of Theorem 4, where, among other variables, the clause $C_{1}$ contains the variable $x_{1}$, the clause $C_{2}$ contains the variables $x_{1}$ and $x_{2}$, and the clause $C_{m}$ contains the variables $x_{2}$ and $x_{n}$.

Proof. Since the number of rounds is $\lceil|V(G)| / 2\rceil$ and there are at most $|V(G)|$ possible moves for a player in any round, the problem is in PSPACE. To prove it is PSPACE-hard, we give a reduction from POS CNF. By adding a dummy variable, POS CNF remains PSPACE-hard if the number of variables $n$ is odd. From an instance $\phi$ of POS CNF where $n$ is odd, we construct, in polynomial time, an instance $G$ of the largest connected subgraph game such that Alice wins in $\phi$ if and only if $G$ is $A$-win. Let $x_{1}, \ldots, x_{n}$ be the variables and let $C_{1}, \ldots, C_{m}$ be the clauses in $\phi$. The construction of $G$ is as follows (see Figure 1 for an illustration): for each variable $x_{i}(1 \leq i \leq n)$, there is a vertex $x_{i}$, and for each clause $C_{j}(1 \leq j \leq m)$, there are 6 vertices $C_{j}^{1}, \ldots, C_{j}^{6}$. For all $1 \leq i \leq n$ and $1 \leq j \leq m$, if the variable $x_{i}$ appears in the clause $C_{j}$, then there is the edge $x_{i} C_{j}^{q}$ for all $1 \leq q \leq 6$. Also, there are the vertices $u, v_{1}, v_{2}, w_{1}, w_{2}$, and $y_{1}, \ldots, y_{n+6 m-2}$, and the edges $w_{1} v_{1}, v_{1} u, u v_{2}$, and $v_{2} w_{2}$. Lastly, for all $1 \leq i \leq n$, there is the edge $u x_{i}$, and, for all $1 \leq \ell \leq n+6 m-2$, there are the edges $w_{1} y_{\ell}$ and $w_{2} y_{\ell}$. To simplify the proof, let $P$ be the subgraph of $G$ induced by the vertices $x_{i}(1 \leq i \leq n)$ and $C_{j}^{q}(1 \leq q \leq 6$ and $1 \leq j \leq m)$, and let $Q$ be the subgraph of $G$ induced by the vertices $V(G) \backslash(V(P) \cup\{u\})$.

First, we prove that, if Alice wins in $\phi$, then $G$ is $A$-win. We give a winning strategy for Alice. In what follows, whenever Alice cannot follow her strategy, she colours an arbitrary vertex and resumes her strategy for the subsequent rounds. Alice first colours $u$. Now, Bob can only construct connected blue subgraphs in $P$ or $Q$ since $u$ separates them. For all $1 \leq j \leq m$, whenever Bob colours a vertex in $\left\{C_{j}^{1}, \ldots, C_{j}^{6}\right\}$, then Alice also colours a vertex in $\left\{C_{j}^{1}, \ldots, C_{j}^{6}\right\}$, so in what follows, we assume that Bob does not colour such a vertex. There are two cases depending on Bob's next move.

Case 1: Bob colours a vertex in $Q$. Then, Alice colours the vertex $x_{i}$ that corresponds to the variable $x_{i}$ she wants to set to true in her winning strategy in $\phi$. Now, whenever Bob colours a vertex $x_{p}(1 \leq p \leq n$ and $p \neq i)$, Alice assumes Bob set the variable $x_{p}$ to false in $\phi$ and colours the vertex in $\left\{x_{1}, \ldots, x_{n}\right\}$ corresponding to her winning strategy in $\phi$. Otherwise, whenever Bob colours a vertex in $Q$, then Alice colours a vertex in $Q$. Note that, by this strategy, Alice ensures a connected red subgraph of order at least $\lceil n / 2\rceil+3 m+1$ since she colours half the variable vertices (rounded up), half the clause vertices, and $u$, 
and since she followed a winning strategy in $\phi$, this subgraph is indeed connected. Furthermore, she ensures that any connected blue subgraph in $P$ is of order at most $\lfloor n / 2\rfloor+3 m$, and hence, Bob must construct his largest connected blue subgraph in $Q$ if he wants to manage a draw. Also note that, if Alice colours $v_{1}$ or $v_{2}$ she wins, since then she ensures a connected red subgraph of order at least $\lceil n / 2\rceil+3 m+2$, while she ensures that any connected blue subgraph in $Q$ is of order at most $\lfloor(n+6 m-2+3-2) / 2\rfloor+2=\lfloor(n-1) / 2\rfloor+3 m+2$. Thus, Bob must have coloured $v_{1}$ and $v_{2}$ in the first two rounds. Now, Alice colours $w_{2}$, and she wins since she ensures that any connected blue subgraph in $Q$ is of order at most $\lfloor(n+6 m-2+2-2) / 2\rfloor+2=\lfloor n / 2\rfloor+3 m+1$.

Case 2: Bob colours a vertex in $\left\{x_{1}, \ldots, x_{n}\right\}$. Then, Alice colours $w_{2}$. This forces Bob to colour $v_{2}$, as otherwise, Alice will colour $v_{2}$ in the next round and win with the following strategy: whenever Bob colours a vertex

- in $\left\{w_{1}, v_{1}\right\}$, then Alice colours the other vertex in $\left\{w_{1}, v_{1}\right\}$;

- $y_{\ell}$, then Alice colours a vertex $y_{k}(\ell \neq k)$;

- $x_{i}(1 \leq i \leq n)$, then Alice colours a vertex $x_{p}(1 \leq p \leq n$ and $i \neq p)$.

In this way, Alice guarantees a connected red subgraph of order at least $\lceil(n+6 m-2+n-3) / 2\rceil+3=n+3 m+1$ without counting any of the vertices $C_{j}^{q}(1 \leq q \leq 6,1 \leq j \leq m)$. Regarding Bob, any connected blue subgraph in $P$ has at most $\lfloor(n-3) / 2\rfloor+3+3 m=\lfloor(n-1) / 2\rfloor+3 m+2$ vertices, and any connected blue subgraph in $Q$ has at most $\lfloor(n+6 m-2+2-3) / 2\rfloor+3=\lfloor(n-1) / 2\rfloor+3 m+2$ vertices. Hence, Alice wins in this case, and thus, we can assume Bob colours $v_{2}$. Now, Alice colours $w_{1}$ and Bob is forced to colour $v_{1}$ for the same reasons as above. Alice now colours $y_{1}$ and then she follows the strategy just previously described above (as in the case where Bob did not colour $v_{2}$ ). In this way, Alice ensures a connected red subgraph of order at least $\lceil(n+6 m-2+2-2) / 2\rceil+2=$ $\lceil n / 2\rceil+3 m+1$ in $Q$. Regarding Bob, any connected blue subgraph in $P$ has at most $\lfloor(n-2) / 2\rfloor+2+3 m=\lfloor n / 2\rfloor+3 m+1$ vertices, and any connected blue subgraph in $Q$ has at most one vertex. Hence, Alice wins in this case as well (recall that $n$ is odd), and this concludes the proof of the first direction.

Now, we prove that if Bob wins in $\phi$, then $G$ is $A B$-draw. We give a drawing strategy for Bob. In what follows, whenever Bob cannot follow his strategy, he colours an arbitrary vertex and resumes his strategy for the subsequent moves of Alice. Part of Bob's strategy is as follows: whenever Alice colours a vertex

- in $\left\{C_{j}^{1}, \ldots, C_{j}^{6}\right\}$ for $1 \leq j \leq m$, then Bob also colours a vertex in $\left\{C_{j}^{1}, \ldots, C_{j}^{6}\right\}$;

- $x_{i}$ for $1 \leq i \leq n$, then Bob assumes Alice set $x_{i}$ to true in $\phi$ and colours the vertex in $\left\{x_{1}, \ldots, x_{n}\right\}$ corresponding to his winning strategy in $\phi$.

So, we just need to give a strategy for Bob in $Q^{\prime}$, the subgraph of $G$ induced by $V(Q) \cup\{u\}$. W.l.o.g., we may assume that the first vertex Alice colours in $Q^{\prime}$ is not $v_{2}$ nor $w_{2}$. Bob colours $w_{2}$. If the first two vertices Alice colours in $Q^{\prime}$ are:

- $w_{1}$ and $v_{1}$, then Bob colours $u$. Now, Alice must colour $v_{2}$, as otherwise, Bob wins as in the proof of the first direction where Alice wins if she manages to 
colour $w_{2}, v_{2}$, and $u$. Then, Bob colours $y_{k}$ for some $1 \leq k \leq n+6 m-2$. Now, whenever Alice colours a vertex $y_{\ell}(1 \leq \ell \leq n+6 m-2)$, then Bob colours a vertex $y_{k}(1 \leq k \leq n+6 m-2$ and $\ell \neq k)$;

$-w_{1}$ and $v_{2}$, then Bob colours $y_{\ell}$ for some $1 \leq \ell \leq n+6 m-2$. Now, whenever Alice colours a vertex in $\left\{v_{1}, u\right\}$, then Bob colours the other vertex in $\left\{v_{1}, u\right\}$. Otherwise, whenever Alice colours a vertex $y_{\ell}(1 \leq \ell \leq n+6 m-2)$, then Bob colours a vertex $y_{k}(1 \leq k \leq n+6 m-2$ and $\ell \neq k)$;

$-w_{1}$ and $u$, then Bob colours $v_{1}$. Now, whenever Alice colours a vertex in $\left\{y_{1}, \ldots, y_{n+6 m-2}, v_{2}\right\}$, Bob colours another vertex in $\left\{y_{1}, \ldots, y_{n+6 m-2}, v_{2}\right\}$;

- $w_{1}$ and $y_{k}$ for some $1 \leq k \leq n+6 m-2$, then Bob colours $v_{2}$. Now, Alice must colour $u$, as otherwise, Bob wins as in the proof of the first direction where Alice wins if she manages to colour $w_{2}, v_{2}$, and $u$. Then, Bob colours $v_{1}$. Now, whenever Alice colours a vertex $y_{\ell}(1 \leq \ell \leq n+6 m-2)$, then Bob colours a vertex $y_{p}(1 \leq p \leq n+6 m-2$ and $\ell \neq p)$;

- any other combination, then Bob colours $w_{1}$. Now, whenever Alice colours a vertex in $\left\{y_{1}, \ldots, y_{n+6 m-2}, v_{1}, v_{2}, u\right\}$, then Bob colours a different vertex in $\left\{y_{1}, \ldots, y_{n+6 m-2}, v_{1}, v_{2}\right\}$ (note that $u$ is not included here).

In the first two cases above, there is a connected blue component in $Q$ of order at least $\lfloor(n+6 m-2+1-2) / 2\rfloor+2=\lfloor(n-1) / 2\rfloor+3 m+1$. In the third case above, there is a connected blue component in $Q$ of order at least $\lfloor(n+6 m-2+1) / 2\rfloor+$ $1=\lfloor(n-1) / 2\rfloor+3 m+1$. In the fourth case above, there is a connected blue component in $Q$ of order at least $\lfloor(n+6 m-2+2-3) / 2\rfloor+2=\lfloor(n-1) / 2\rfloor+3 m+1$. In the last case above, there is a connected blue component in $Q$ of order at least $\lfloor(n+6 m-2+4-4) / 2\rfloor+2=\lfloor n / 2\rfloor+3 m+1=\lfloor(n-1) / 2\rfloor+3 m+1$ (since $n$ is odd). To summarise, in each of the cases, Bob has ensured that there is a connected blue component in $Q$ of order at least $\lfloor(n-1) / 2\rfloor+3 m+1$.

Regarding Alice, in the first two cases above, any connected red component in $Q$ is of order at most $\lceil(n+6 m-2+2-3) / 2\rceil+2=\lceil(n-1) / 2\rceil+3 m+1$. In the third case above, any connected red component in $Q$ is of order at most $\lceil(n+6 m-2+1-1) / 2\rceil+1=\lceil n / 2\rceil+3 m=\lceil(n-1) / 2\rceil+3 m+1$ (since $n$ is odd). In the fourth case above, any connected red component in $Q$ is of order at most $\lceil(n+6 m-2+1-2) / 2\rceil+2=\lceil(n-1) / 2\rceil+3 m+1$. In the last case above, any connected red component in $Q$ is of order at most 1 . Thus, in each of the cases, Bob ensured that any connected red component in $Q$ is of order at most $\lceil(n-1) / 2\rceil+3 m+1=\lfloor(n-1) / 2\rfloor+3 m+1$ (since $n$ is odd). Hence, for Alice to win, she must have a connected red component of order at least $\lfloor(n-1) / 2\rfloor+3 m+2$ in $P^{\prime}$, the subgraph of $G$ induced by $V(P) \cup\left\{u, v_{1}, v_{2}\right\}$ (since, by Bob's strategy, it can never be that $u, v_{1}$, and $w_{1}\left(u, v_{2}\right.$, and $w_{2}$, resp.) are all red). Since Bob follows a winning strategy in $\phi$ whenever Alice colours a vertex in $\left\{x_{1}, \ldots, x_{n}\right\}$, there is a $j$ for which no vertex in $C_{j}^{1}, \ldots, C_{j}^{6}$ is adjacent to a red vertex. Thus, any connected red component in $P^{\prime}$ has order at most $\lceil(n+6 m-6) / 2\rceil+3=\lceil n / 2\rceil+3 m=\lfloor(n-1) / 2\rfloor+3 m+1$ (since $n$ is odd). Thus, in $G$, there is a connected blue component of order at least $\lfloor(n-1) / 2\rfloor+3 m+1$ and any connected red component has order at most $\lfloor(n-1) / 2\rfloor+3 m+1$, so Alice does not win in any of the cases. This ends the proof of the second direction. 


\section{Paths and Cycles}

In this section, we deal with the case of $n$-vertex paths $P_{n}=\left(v_{1}, \ldots, v_{n}\right)$ and cycles $C_{n}=\left(v_{1}, \ldots, v_{n}\right)$. We begin with two lemmas for specific cases in paths, which we use in the proofs for paths and cycles of odd order. In the following proofs in this section, we often divide the main path $P_{n}$ into two subpaths $Q$ and $Q^{\prime}$, and say that Alice "follows" Bob, that is, when Bob plays in $Q$ (in $Q^{\prime}$, resp.), Alice then plays in $Q$ (in $Q^{\prime}$, resp.). The way Alice answers to Bob's moves in $Q$ (in $Q^{\prime}$, resp.) is given in the proofs and depends on the different cases. Note that, when following this strategy, Alice may be unable to colour a desired vertex (because $Q$, resp., $Q^{\prime}$, has no uncoloured vertex anymore, or because the desired vertex is already red). In this case, Alice colours an arbitrary uncoloured vertex of $P_{n}$. The same applies for when we say that Bob "follows" Alice.

Lemma 1. [2] For all $n \geq 1$, for the path $P_{n}$, Bob has a strategy that ensures that the largest connected red subgraph is of order at most 2 , even if one of the path's vertices of degree 1 is initially coloured red and it is Alice's turn.

Lemma 2. [2] Let $x \geq 1$ and $n \geq x$. For any path $P_{n}$ with $x$ vertices initially coloured blue, let $y$ be the maximum order of an initial connected blue component.

- if $y=x$ and, either the blue component contains no ends of $P_{n}$ or $x=1$, then, if Alice starts, she has a strategy ensuring that Bob cannot create a connected blue component of order more than $x+1$;

- otherwise, if Alice starts, she has a strategy ensuring that Bob cannot create a connected blue component of order more than $x$.

Theorem 5. For all $n \geq 1$, the path $P_{n}$ is $A$-win if and only if $n \in\{1,3,5,7,9\}$.

Proof. By Theorem 1, we must prove that $P_{n}$ is $A$-win if $n \in\{1,3,5,7,9\}$, and $P_{n}$ is $A B$-draw otherwise. By Theorem 2, $P_{n}$ is $A B$-draw if $n$ is even. If $n \leq 9$ is odd, by a case analysis, Alice has a winning strategy that first colours the center of $P_{n}$. So, let us assume that $n \geq 11$ is odd. We orient the path from left to right (from $v_{1}$ to $v_{n}$ ), so we can use the notions of left and right. We now give a drawing strategy for Bob when $n \geq 11$. Let $v_{j}(1 \leq j \leq n)$ be the first vertex coloured by Alice. Since $n \geq 11$, there are at least 5 vertices to the left or right of $v_{j}$, say to the left of $v_{j}$, i.e., $5 \leq j \leq n$. Bob colours $v_{j-1}$. Let $Q=\left(v_{1}, \ldots, v_{j-1}\right)$ and $Q^{\prime}=\left(v_{j}, \ldots, v_{n}\right)$. Now, Bob "follows" Alice, that is, when Alice plays in $Q\left(Q^{\prime}\right.$, resp.), Bob then plays in $Q\left(Q^{\prime}\right.$, resp.), and both games are considered independently (since $v_{j-1}$ is blue and $v_{j}$ is red). Considering $Q^{\prime}$ as a path with one of its ends initially coloured red, and applying Lemma 1 to it, Bob can ensure that Alice cannot create a connected red component of order more than 2 in $Q^{\prime}$. Let $v_{\ell}$ be the first vertex that Alice colours in $Q$. We distinguish two cases:

Case 1: $\ell \neq j-2$. Bob colours $v_{j-2}$. Now, whenever Alice plays in $Q$, while it is possible, Bob colours a neighbour of the connected blue component containing $v_{j-1}$ and $v_{j-2}$. If it is not possible anymore, either the connected blue component is of order $\lceil(j-1) / 2\rceil \geq 2$ (in which case the largest connected red component 
in $Q$ is of order $\lfloor(j-1) / 2\rfloor$ and so, the game is a draw) or it is of order $2 \leq x<$ $(j-1) / 2$ and it is Bob's turn. In the latter case, the connected blue component in $Q$ consists of the vertices $v_{j-x}, \ldots, v_{j-1}$, and $v_{j-x-1}$ is red since Bob cannot colour a neighbour of the connected blue component. Let $R=\left(v_{1}, \ldots, v_{j-x-1}\right)$ and note that there are exactly $x$ red vertices in $R$ including $v_{j-x-1}$ (one of its ends). Then, applying Lemma 2 to $R$ (but with Bob as the first player), Bob has a strategy ensuring that Alice cannot create a connected red component of order more than $x$ in $R$. Hence, the game in $P_{n}$ ends in a draw in this case.

Case 2: $\ell=j-2$. Bob colours $v_{j-4}$. If Alice colours $v_{j-3}$, then Bob colours $v_{j-5}$, and vice versa, and this ensures a connected blue component of order at least 2 . Otherwise, if Alice colours a vertex $v_{t}$ with $1 \leq t \leq j-6$, then Bob colours $v_{t+1}$, unless $v_{t+1}$ is already coloured, in which case, Bob colours $v_{t-1}$. In the latter case, Bob can ensure a draw since he can ensure that Alice cannot create a connected red component of order more than 2 in $R^{*}=\left(v_{1}, \ldots, v_{t-1}\right)$ by Lemma 1 . So, assume we are in the former case. Let $R=\left(v_{1}, \ldots, v_{t}\right)$ and $R^{\prime}=\left(v_{t+1}, \ldots, v_{j-5}\right)$. From now on, Bob "follows" Alice (unless Alice colours $v_{j-5}$, in which case, Bob colours $v_{j-3}$ ), that is, when Alice plays in $R$ (in $R^{\prime}$, resp.), Bob then plays in $R$ (in $R^{\prime}$, resp.), and both games are considered independently (since $v_{t}$ is coloured red and $v_{t+1}$ is coloured blue). Considering $R$ as a path with one of its ends initially coloured red, and applying Lemma 1 to it, Bob has a strategy ensuring that Alice cannot create a connected red component of order more than 2 in $R$. Bob plays in $R^{\prime}$ assuming that $v_{j-5}$ is already coloured red, and applying Lemma 1 to it, Bob has a strategy ensuring that Alice cannot create a connected red component of order more than 2 in $R^{\prime}$. It is easy to see that, in this case, the largest connected blue (red, resp.) subgraph is of order 2 (at most 2, resp.).

Now, we address the largest connected subgraph game in cycles. We start with a lemma for a specific case in paths, which we use in the proof for cycles.

Lemma 3. [2] Let $x \geq 3, n \geq x+1$, and $n-x$ be odd. For any path $P_{n}$ with $x$ vertices, including both ends, initially blue, if Alice starts, then she can ensure that no connected blue component of order more than $x-1$ is created in $P_{n}$.

Theorem 6. For all $n \geq 3$, the cycle $C_{n}$ is $A$-win if and only if $n$ is odd.

Proof. If $n$ is even, then $C_{n}$ is a reflection graph, and thus, is $A B$-draw by Theorem 2. So let $n$ be odd. We describe a winning strategy for Alice. If $n \leq 5$, the result is obvious, so let us assume that $n>5$. First, let us assume (independently of how this configuration appears) that after $x \geq 3$ rounds, the vertices $v_{1}, \ldots, v_{x}$ are red, the vertices $v_{n}$ and $v_{x+1}$ are blue, and any $x-2$ other vertices in $\left\{v_{x+2}, \ldots, v_{n-1}\right\}$ are blue. Note that it is Alice's turn. By Lemma 3, Alice may ensure that Bob cannot create a connected blue component of order at least $x$ in the subgraph induced by $\left(v_{x+1}, \ldots, v_{n}\right)$. Thus, in this case, Alice wins.

Now, let Alice first colour the vertex $v_{1}$. If Bob does not colour a neighbour of $v_{1}$ (say Bob colours $v_{j}$ with $3<j<n$, since $n \geq 5$ and odd), then, on her second turn, Alice colours $v_{2}$. Then, while it is possible, Alice colours a neighbour of the 
connected red component. When it is not possible anymore, either the connected red component is of order $\lceil n / 2\rceil$ or it is of order at least 3 and we are in the situation of the above paragraph. In both cases, Alice wins.

Therefore, after Alice colours her first vertex (call it $v_{2}$ ), Bob must colour some neighbour of it (say $v_{1}$ ). By induction on the number $t \geq 1$ of rounds, let us assume that the game reaches, after $t$ rounds, a configuration where, for every $1 \leq i \leq t$, vertices $v_{2 i-1}$ are coloured blue and vertices $v_{2 i}$ are coloured red. If $t=\lfloor n / 2\rfloor$, then Alice finally colours $v_{n}$ (recall that $n$ is odd) and wins. Otherwise, let Alice colour $v_{2 t+2}$. If Bob then colours $v_{2 t+1}$, then we are back to the previous situation for $t^{\prime}=t+1$. Then, eventually, Alice wins by induction on $n-2 t$. If Bob does not colour $v_{2 t+1}$, then Alice colours $v_{2 t+1}$ and then continues to grow the connected red component containing $v_{2 t+1}$ while possible. When it is not possible anymore, note that removing (or contracting) the vertices $v_{2}$ to $v_{2 t}$, we are back to the situation of the first paragraph of this proof (with a connected red component of order at least 3) and, therefore, Alice wins.

\section{Cographs}

For paths and cycles, optimal play depended on positional play with respect to the previously coloured vertices since the graphs are sparse, making it easy for the players to stop the expansion of the opponent's largest connected component. As a consequence, in such cases, players must stop growing their largest connected component, and start growing a new one. Such a strategy is likely to be less viable in denser graphs, in which the game tends to turn into a different one, where the players grow a single connected component each, that they have to keep "alive" for as long as possible. We illustrate this with the case of cographs, which leads us to introduce a few more notations (see $\mathcal{A}^{*}$ below) to describe a linear-time algorithm deciding the outcome of the game in such instances.

A graph $G$ is a cograph if it is $P_{4}$-free, i.e., it does not contain $P_{4}$ as an induced subgraph. The class of cographs can be defined recursively as follows. The single-vertex graph $K_{1}$ is a cograph. Let $G_{1}$ and $G_{2}$ be two cographs. Then, the disjoint union $G_{1}+G_{2}$ is a cograph. Moreover, the join $G_{1} \oplus G_{2}$, obtained from $G_{1}+G_{2}$ by adding all the possible edges between the vertices of $G_{1}$ and $G_{2}$, is a cograph. Recall that a decomposition, i.e., a sequence of disjoint unions and joins from single vertices, of a cograph can be computed in linear time [6].

To simplify notation in Theorem 7 and its proof, let $\mathcal{A}^{*}$ be the set of graphs such that there exists a strategy for Alice that ensures a connected red component of order $\lceil|V(G)| / 2\rceil$, regardless of Bob's strategy. I.e., $\mathcal{A}^{*}$ is the set of graphs in which Alice has a strategy to ensure a single connected red component.

Theorem 7. Let $G$ be a cograph. There exists a linear-time algorithm that decides whether $G$ is $A$-win or $A B$-draw, and whether $G \in \mathcal{A}^{*}$ or not.

Proof. The proof is by induction on $n=|V(G)|$. More precisely, we describe a recursive algorithm. If $n=1$, then $G$ is clearly $A$-win and $G \in \mathcal{A}^{*}$. 
Let us assume that $n>1$. There are two cases to be considered. Either $G=G_{1} \oplus G_{2}$ for some cographs $G_{1}$ and $G_{2}$, or $G=G_{1}+G_{2}+\ldots, G_{m}$, where, for every $1 \leq i \leq m(m \geq 2), G_{i}$ is either a single vertex or is a cograph obtained from the join of two other cographs. For every $1 \leq i \leq m$, let us assume by induction that it can be computed in time linear in $\left|V\left(G_{i}\right)\right|$, whether $G_{i}$ is $A$-win or $A B$-draw and whether $G_{i} \in \mathcal{A}^{*}$ or not. Let us show how to decide if $G$ is $A$-win or $A B$-draw, and whether $G \in \mathcal{A}^{*}$ or not, in constant time.

1. Let us first assume that $\boldsymbol{G}=\boldsymbol{G}_{\mathbf{1}} \oplus \boldsymbol{G}_{\mathbf{2}}$. We prove that (see [2]):

(a) If $n$ is odd, then $G$ is $A$-win and $G \in \mathcal{A}^{*}$.

(b) If $\left|V\left(G_{1}\right)\right|,\left|V\left(G_{2}\right)\right| \geq 2$ and $n$ is even, then $G$ is $A B$-draw and $G \in \mathcal{A}^{*}$.

(c) If $\left|V\left(G_{1}\right)\right|=1$ and $n$ is even, there are two cases to consider:

i. If $G_{2} \notin \mathcal{A}^{*}$, then $G$ is $A$-win and $G \in \mathcal{A}^{*}$.

ii. If $G_{2} \in \mathcal{A}^{*}$, then $G$ is $A B$-draw and $G \in \mathcal{A}^{*}$.

2. Now, let us assume that $\boldsymbol{G}=\boldsymbol{G}_{\mathbf{1}}+\boldsymbol{G}_{\mathbf{2}}+\ldots+\boldsymbol{G}_{\boldsymbol{m}}$ where, for every $1 \leq$ $i \leq m(m \geq 2), G_{i}$ is either a single vertex or a cograph obtained from the join of two cographs $G_{i}^{\prime}$ and $G_{i}^{\prime \prime}$ such that $\left|V\left(G_{i}^{\prime}\right)\right| \geq\left|V\left(G_{i}^{\prime \prime}\right)\right|$. Also, let $n_{i}=$ $\left|V\left(G_{i}\right)\right|$ for every $1 \leq i \leq m$, and let us assume that $n_{1} \geq n_{2} \geq \cdots \geq n_{m}$.

To simplify the proof to follow, first note that, if $n_{1}=1$, then $G$ is $A B$-draw (since $n_{2}=1$ as $m \geq 2$ ) and $G \in \mathcal{A}^{*}$ if and only if $G=G_{1}+G_{2}$. Second, if $n_{2}=1$, then the result of the game in $G$ is the same as the result of the game in $G_{1}$, which is known, by Case 1 , since $G_{1}$ is a join. Moreover, in this case, $G \in \mathcal{A}^{*}$ if and only if $n_{1}$ is odd and $G=G_{1}+G_{2}$. Hence, we may assume that $n_{1}>1$ and $n_{2}>1$. Lastly, in what follows, for any of the winning strategies described for Alice, whenever Bob colours a vertex in $G_{j}$ for $3 \leq j \leq m$, Alice also colours a vertex in $G_{j}$ on her next turn. The same holds for any of the drawing strategies for Bob (with Bob and Alice reversed), except for Case 2(e)ii, for which the same only holds for $4 \leq j \leq m$. This guarantees that a player never has a connected component of order more than $\left\lceil\frac{n_{j}}{2}\right\rceil$ in $G_{j}$ for $3 \leq j \leq m(4 \leq j \leq m$ for Case 2(e)ii). Alice always has a connected red component of order at least $\left\lceil\frac{n_{1}}{2}\right\rceil$ in all of the winning strategies of Alice below, and Bob always has a connected blue component of order at least $\left\lceil\frac{n_{1}}{2}\right\rceil$ in all of the drawing strategies of Bob below. Hence, for all the cases except Case 2(e)ii, we can assume that $G=G_{1}+G_{2}$, and for Case 2(e)ii, we can assume that $G=G_{1}+G_{2}+G_{3}$. In what follows, if a player cannot follow their strategy in a round, unless otherwise stated, they colour an arbitrary vertex and then resume their strategy for the subsequent rounds.

There are 5 cases to consider, and recall that we assume that $n_{1}>1$ and $n_{2}>1$, so $G_{1}^{\prime \prime}$ and $G_{2}^{\prime \prime}$ exist. In Case 2(e)iii below, the statement involves $n_{3}$, so if $m=2$, then we consider that $n_{3}=0$. Also, since Bob always has a strategy where, for each $1 \leq i \leq m$, he colours at least $\left\lfloor\frac{n_{i}}{2}\right\rfloor$ vertices of $G_{i}$ blue, and since $n_{2}>1$, then $G \notin \mathcal{A}^{*}$ in all of the following cases. Thus, we just need to show the outcome of the game on $G$ for each case.

(a) If $n_{1}=n_{2}$, then $G$ is $A B$-draw.

Assume, w.l.o.g., that Alice first colours a vertex in $G_{1}$. Bob then colours a vertex in $G_{2}^{\prime \prime}$. Then, whenever Alice colours a vertex in $G_{1}\left(G_{2}\right.$, resp.), 
Bob also colours a vertex in $G_{1}\left(G_{2}\right.$, resp.). In particular, if Bob is to colour a vertex in $G_{2}$, then he colours one in $G_{2}^{\prime}$ first if possible, if not, then he colours a vertex in $G_{2}^{\prime \prime}$, and, if that is not possible, he colours a vertex in $G_{1}$. Similarly, if Bob is to colour a vertex in $G_{1}$ by this strategy, but cannot since all of the vertices of $G_{1}$ are coloured, then he colours one in $G_{2}^{\prime}$ first if possible, and if not, then he colours a vertex in $G_{2}^{\prime \prime}$.

If $n_{1}$ is odd, then by this strategy, Bob ensures a connected blue component of order $\frac{n_{2}-1}{2}+1=\frac{n_{1}-1}{2}+1$ in $G_{2}$ and that the largest connected red component in $G$ is of order at most $\frac{n_{1}-1}{2}+1$. If $n_{1}$ is even, then by this strategy, if Alice colours the last vertex in $G_{1}$, then Bob ensures a connected blue component of order $\left\lceil\frac{n_{2}-1}{2}\right\rceil+1=\left\lceil\frac{n_{1}-1}{2}\right\rceil+1$ in $G_{2}$ and that the largest connected red component in $G$ is of order at most $\left\lceil\frac{n_{1}-1}{2}\right\rceil+1$. If Alice did not colour the last vertex in $G_{1}$, and so, she coloured the last vertex in $G_{2}$, then Bob ensures a connected blue component of order $\left\lceil\frac{n_{2}-2}{2}\right\rceil+1=\frac{n_{1}}{2}$ in $G_{2}$ and that the largest connected red component in $G$ is of order at most $\frac{n_{1}}{2}$. Hence, $G$ is $A B$-draw.

(b) If $n_{1}>n_{2}$ and $n_{1}$ is odd, then $G$ is $A$-win.

Alice first colours a vertex in $G_{1}$. Then, whenever Bob colours a vertex in $G_{1}\left(G_{2}\right.$, resp.), Alice colours a vertex in $G_{1}\left(G_{2}\right.$, resp.). By Case 1(a), Alice has a winning strategy in $G_{1}$ ensuring a connected red component of order at least $\left\lceil\frac{n_{1}}{2}\right\rceil$. By Case 1, Alice ensures that any connected blue component in $G_{2}$ is of order at most $\left\lceil\frac{n_{2}}{2}\right\rceil<\left\lceil\frac{n_{1}}{2}\right\rceil$. Hence, $G$ is $A$-win.

(c) If $n_{1}>n_{2}, n_{1}$ is even, and $\left|V\left(G_{1}^{\prime \prime}\right)\right| \geq 2$, then $G$ is $A B$-draw.

Whenever Alice colours a vertex in $G_{1}\left(G_{2}\right.$, resp.), Bob also colours a vertex in $G_{1}\left(G_{2}\right.$, resp.). By Case 1(b), Bob has a drawing strategy in $G_{1}$ ensuring a connected blue component of order at least $\frac{n_{1}}{2}$. By Case 1 , Bob ensures that any connected red component in $G_{2}$ is of order at most $\left\lceil\frac{n_{2}}{2}\right\rceil \leq \frac{n_{1}}{2}$. Hence, $G$ is $A B$-draw.

(d) If $n_{1}>n_{2}, n_{1}$ is even, $\left|V\left(G_{1}^{\prime \prime}\right)\right|=1$, and $G_{1}^{\prime} \in \mathcal{A}^{*}$, then $G$ is $A B$-draw. Whenever Alice colours a vertex in $G_{1}\left(G_{2}\right.$, resp.), Bob also colours a vertex in $G_{1}\left(G_{2}\right.$, resp.). By Case 1(c)ii, Bob has a drawing strategy in $G_{1}$ ensuring a connected blue component of order at least $\frac{n_{1}}{2}$. By Case 1, Bob ensures that any connected red component in $G_{2}$ is of order at most $\left\lceil\frac{n_{2}}{2}\right\rceil \leq \frac{n_{1}}{2}$. Hence, $G$ is $A B$-draw.

(e) If $n_{1}>n_{2}, n_{1}$ is even, $\left|V\left(G_{1}^{\prime \prime}\right)\right|=1$, and $G_{1}^{\prime} \notin \mathcal{A}^{*}$, then:

i. If $n_{1}>n_{2}+1$, then $G$ is $A$-win.

Alice first colours a vertex in $G_{1}$. Then, whenever Bob colours a vertex in $G_{1}\left(G_{2}\right.$, resp.), Alice colours a vertex in $G_{1}\left(G_{2}\right.$, resp.). By Case 1(c)i, Alice ensures a connected red component of order at least $\frac{n_{1}}{2}$ in $G_{1}$, and that any connected blue component in $G_{1}$ is of order less than $\frac{n_{1}}{2}$. By Case 1, Alice ensures that any connected blue component in $G_{2}$ is of order at most $\left\lceil\frac{n_{2}}{2}\right\rceil<\frac{n_{1}}{2}$. Hence, $G$ is $A$-win. ii. If $n_{1}=n_{2}+1=n_{3}+1$, then $G$ is $A B$-draw.

Whenever Alice colours a vertex in $G_{1}$, Bob colours a vertex in $G_{1}$. By Case 1, this ensures that $\frac{n_{1}}{2}$ of the vertices in $G_{1}$ are red and $\frac{n_{1}}{2}$ are blue. The first time that Alice colours a vertex $v \in V\left(G_{2}\right) \cup$ 
$V\left(G_{3}\right)$, assume, w.l.o.g., that $v \in V\left(G_{2}\right)$. Bob then colours a vertex in $G_{3}^{\prime \prime}$. Then, whenever Alice colours a vertex in $G_{2}\left(G_{3}\right.$, resp.), Bob colours a vertex in $G_{2}\left(G_{3}\right.$, resp.). In particular, if Bob is to colour a vertex in $G_{3}$, then he colours one in $G_{3}^{\prime}$ first if possible, if not, then he colours a vertex in $G_{3}^{\prime \prime}$, and, if that is not possible, he colours a vertex in $G_{2}$. As in Case 2(a), Bob ensures a connected blue component of order $\left\lceil\frac{n_{3}}{2}\right\rceil=\frac{n_{1}}{2}$ in $G_{3}$ and that any connected red component in $G_{2}$ is of order at most $\left\lceil\frac{n_{2}}{2}\right\rceil=\frac{n_{1}}{2}$. Hence, $G$ is $A B$-draw.

iii. If $n_{1}=n_{2}+1$ and $n_{2}>n_{3}$, then $G$ is $A$-win.

Alice first colours the vertex in $G_{1}^{\prime \prime}$. Then, Alice colours vertices in $G_{1}$ as long as she can. By Case 1(c)i, she ensures that any connected blue component in $G_{1}$ is of order less than $\frac{n_{1}}{2}$. If it is Alice's turn, there is a connected red component of order $n_{1}-k$ in $G_{1}$ for some $0 \leq k \leq \frac{n_{1}}{2}$, and it is the first round in which she can no longer colour vertices in $G_{1}$, then Bob coloured $k$ vertices in $G_{1}$ and $n_{1}-2 k$ vertices in $G_{2}$. Then, any connected blue component in $G_{2}$ is of order at most $\left\lceil\frac{n_{2}-n_{1}+2 k-1}{2}\right\rceil+n_{1}-2 k=n_{1}-k-1<n_{1}-k$. Hence, $G$ is $A$-win.

We get the result as a decomposition of a cograph is computed in linear time.

\section{Further Work}

It would be interesting to study the game in other graph classes such as trees and interval graphs. Also, since grids of even order are $A B$-draw by Theorem 2, it would be intriguing to look at grids of odd order. Just as reflection graphs are a large class of graphs that are $A B$-draw, another direction would be to find a diverse class of graphs that are $A$-win. Any graph $G \in \mathcal{A}^{*}$ of odd order is $A$-win, and so, perhaps a class of dense graphs of odd order would be a prime candidate.

\section{References}

1. Andres, S.D., Huggan, M., Mc Inerney, F., Nowakowski, R.J.: The orthogonal colouring game. Theoretical Computer Science 795, 312-325 (2019)

2. Bensmail, J., Fioravantes, F., Mc Inerney, F., Nisse, N.: The Largest Connected Subgraph Game. Research report (2021), https://hal.inria.fr/hal-03137305

3. Bonnet, E., Jamain, F., Saffidine, A.: On the complexity of connection games. Theoretical Computer Science 644, 2-28 (2016)

4. Browne, C.: Connection Games: Variations on a Theme. AK Peters (2005)

5. Bruno, J., Weinberg, L.: A constructive graph-theoretic solution of the shannon switching game. IEEE Transactions on Circuit Theory 17(1), 74-81 (1970)

6. Corneil, D.G., Perl, Y., Stewart, L.K.: A linear recognition algorithm for cographs. SIAM Journal on Computing 14(4), 926-934 (1985)

7. Duchêne, É., Gonzalez, S., Parreau, A., Rémila, E., Solal, P.: INFLUENCE: a partizan scoring game on graphs. CoRR abs/2005.12818 (2020)

8. Even, S., Tarjan, R.E.: A combinatorial problem which is complete in polynomial space. J. ACM 23(4), 710-719 (1976) 
9. Gardner, M.: The Scientific American Book of Mathematical Puzzles \& Diversions. Simon and Schuster, N.Y. (1959)

10. Gardner, M.: The Second Scientific American Book of Mathematical Puzzles and Diversions. Simon and Schuster, N.Y. (1961)

11. Larsson, U., Nowakowski, R.J., Neto, J.P., Santos, C.P.: Guaranteed scoring games. The Electronic Journal of Combinatorics 23(3) (2016)

12. Larsson, U., Nowakowski, R.J., Santos, C.P.: Games with guaranteed scores and waiting moves. International Journal of Game Theory 47(2), 653-671 (2018)

13. Micek, P., Walczak, B.: A graph-grabbing game. Comb. Probab. Comput. 20(4), 623-629 (2011)

14. Reisch, S.: Hex ist PSPACE-vollständig. Acta Informatica 15(2), 167-191 (1981)

15. Schaefer, T.J.: On the complexity of some two-person perfect-information games. Journal of Computer and System Sciences 16(2), 185-225 (1978)

16. Shapovalov, A.: Occupation games on graphs in which the second player takes almost all vertices. Discret. Appl. Math. 159(15), 1526-1527 (2011)

\section{Appendix: Omitted proofs}

Here, we give proofs that were sketched or omitted in the main part of the paper, due to lack of space. Recall that a full version of the paper is available [2].

Theorem 1. There does not exist a graph $G$ that is B-win.

Proof. Towards a contradiction, assume there exists a graph $G$ that is $B$-win. Consider the following strategy for Alice. In the first round, Alice colours an arbitrary vertex $v \in V(G)$. Now, one vertex is coloured and it can be assumed that Bob is the first player. Alice now plays according to the second player's winning strategy in $G$. If, by this strategy, Alice is ever required to colour an already-coloured vertex, then that vertex must be red, and again, in this case, Alice colours an arbitrary uncoloured vertex. Since the only reason a vertex cannot be coloured is that it is already coloured, Alice can always follow this strategy, which is a winning strategy, a contradiction.

Theorem 2. Any reflection graph $G$ is $A B$-draw.

Proof. We define a "copying" strategy for Bob which guarantees a draw. Let $U=\left\{u_{1}, \ldots, u_{n}\right\}$ and $V=\left\{v_{1}, \ldots, v_{n}\right\}$ be a partitioning of the vertices of $G$ that satisfies the two conditions required for $G$ to be a reflection graph. Bob's copying strategy is as follows. In every round, when Alice colours a vertex $u_{i} \in U$ $\left(v_{i} \in V\right.$, resp.), Bob colours its image $v_{i} \in V\left(u_{i} \in U\right.$, resp.). By Bob's strategy, it is easy to see that Bob can always play in this way. Moreover, by the symmetry of the graph, for every vertex coloured red (blue, resp.) in $U$, its image is coloured blue (red, resp.) in $V$. Hence, once all vertices are coloured, by the symmetry of the graph and the second condition for reflection graphs concerning the edges between vertices of $U$ and $V$, there is a blue isomorphic copy of any connected red subgraph in $G$. Thus, the game ends in a draw.

Theorem 3. Given a graph $G$, deciding if $G$ is a reflection graph is GI-hard. 
Proof. The reduction is from the GraPh IsOmorPhISm problem, in which, given two input graphs $G_{1}$ and $G_{2}$, one has to decide whether $G_{1}$ and $G_{2}$ are isomorphic. We may further assume that $G_{1}$ and $G_{2}$ are each connected and of odd order, which is one of the input restrictions for which the problem remains hard. Indeed, note that we obtain an equivalent instance of the problem (with the desired properties), upon adding, if needed, one or two universal vertices to both $G_{1}$ and $G_{2}$.

We construct a graph $H$ in polynomial time, such that $G_{1}$ and $G_{2}$ are isomorphic if and only if $H$ is a reflection graph. The graph $H$ we construct is simply $G_{1}+G_{2}$, the disjoint union of $G_{1}$ and $G_{2}$. Let us prove the two directions of the equivalence.

First, we prove the forward direction. Assume that the vertices of $G_{1}$ and $G_{2}$ are $u_{1}, \ldots, u_{n}$ and $v_{1}, \ldots, v_{n}$, respectively, ordered in such a way that there is an isomorphism between $G_{1}$ and $G_{2}$ where $v_{i}$ is the image of $u_{i}$, for all $1 \leq i \leq n$. Note that no edge joins a vertex from $G_{1}$ and a vertex from $G_{2}$. Then, $G_{1} \cup G_{2}=$ $H$ is a reflection graph with $U=V\left(G_{1}\right)$ and $V=V\left(G_{2}\right)$. The reflection property is trivial in that case.

Now, we prove the other direction. Assume that $H$ is a reflection graph with parts $U=\left\{u_{1}, \ldots, u_{n}\right\}$ and $V=\left\{v_{1}, \ldots, v_{n}\right\}$ such that the function mapping $u_{i}$ to $v_{i}$ (for all $1 \leq i \leq n$ ) is an isomorphism between $H[U]$ and $H[V]$. If $U$ is precisely $V\left(G_{1}\right)$ while $V$ is precisely $V\left(G_{2}\right)$, then we get that $H[U]=G_{1}$ and $H[V]=G_{2}$ are isomorphic, by definition of a reflection graph. So, assume this is not the case.

For all $1 \leq i \leq n$, note that either 1) $u_{i} \in V\left(G_{1}\right)$ and $\left.v_{i} \in V\left(G_{2}\right), 2\right)$ $u_{i} \in V\left(G_{2}\right)$ and $\left.v_{i} \in V\left(G_{1}\right), 3\right) u_{i}, v_{i} \in V\left(G_{1}\right)$, or 4) $u_{i}, v_{i} \in V\left(G_{2}\right)$. We consider all $i$ 's in turn, and possibly switch vertices of $U$ and $V$ as follows:

- If $u_{i}$ and $v_{i}$ satisfy Condition 1 ) above, then we do nothing.

- If $u_{i}$ and $v_{i}$ satisfy Condition 2) above, then we move $u_{i}$ from $U$ to $V$, and, conversely, move $v_{i}$ from $V$ to $U$, resulting in a bipartition of $V(H)$ into two parts $U^{\prime}$ and $V^{\prime}$. Note that, considering the ordering $u_{1}^{\prime}, \ldots, u_{n}^{\prime}$ and $v_{1}^{\prime}, \ldots, v_{n}^{\prime}$ of $U^{\prime}$ and $V^{\prime}$ (where $u_{j}^{\prime}=u_{j}$ and $v_{j}^{\prime}=v_{j}$ for all $1 \leq j \leq n$ such that $i \neq j$, and $u_{i}^{\prime}=v_{i}$ and $v_{i}^{\prime}=u_{i}$ ), respectively, we have that $H$ is also a reflection graph with respect to the two parts $U^{\prime}$ and $V^{\prime}$. Indeed, by the isomorphism and reflection properties, we have that $u_{i}$ was neighbouring $u_{i_{1}}, \ldots, u_{i_{k}}$ in $U$ (and so, $v_{i}$ was neighbouring $v_{i_{1}}, \ldots, v_{i_{k}}$ in $V$ ) and $v_{j_{1}}, \ldots, v_{j_{k}}$ in $V$ (and so, $v_{i}$ was neighbouring $u_{j_{1}}, \ldots, u_{j_{k}}$ in $U$ ), which translates, for $U^{\prime}$ and $V^{\prime}$, into $u_{i}^{\prime}$ neighbouring $v_{j_{1}}, \ldots, v_{j_{k}}$ in $V^{\prime}$ (and so, $v_{i}^{\prime}$ neighbouring $u_{j_{1}}, \ldots, u_{j_{k}}$ in $U^{\prime}$ ) and $u_{i_{1}}, \ldots, u_{i_{k}}$ in $U^{\prime}$ (and so, $v_{i}^{\prime}$ neighbouring $v_{i_{1}}, \ldots, v_{i_{k}}$ in $V^{\prime}$ ).

- If $u_{i}$ and $v_{i}$ satisfy Condition 3) or 4) above, then we get a contradiction to one of the original assumptions on $G_{1}$ and $G_{2}$. Indeed, assume, w.l.o.g., that $u_{i}$ and $v_{i}$ satisfy Condition 3 ), i.e., both $u_{i}$ and $v_{i}$ originate from $G_{1}$. Note that, because $G_{1}$ and $G_{2}$ are each connected and of odd order, there must be a pair $u_{j}, v_{j}$ such that, without loss of generality, $u_{j} \in V\left(G_{1}\right)$ and $v_{j} \in V\left(G_{2}\right)$. Furthermore, since $G_{1}$ is connected, for such a pair $u_{j}, v_{j}$, it can be assumed, w.l.o.g., that at least one of $u_{i} u_{j}$ and $v_{i} u_{j}$ is an edge. If 
the former edge exists, then the contradiction arises from the fact that, since $H$ is a reflection graph, we must have the edge $v_{i} v_{j}$ as well, which is not possible since $v_{i} \in V\left(G_{1}\right)$ and $v_{j} \in V\left(G_{2}\right)$. If the latter edge exists, then, because $H$ is a reflection graph, the edge $u_{i} v_{j}$ also exists, hence, an edge between $G_{1}$ and $G_{2}$, which again is a contradiction.

Once all $i$ 's have been treated this way, $H$ remains a reflection graph, and a direct isomorphism between $G_{1}$ and $G_{2}$ is deduced.

Lemma 1. For all $n \geq 1$, for the path $P_{n}$, Bob has a strategy that ensures that the largest connected red subgraph is of order at most 2 , even if one of the path's vertices of degree 1 is initially coloured red and it is Alice's turn.

Proof. Assume, w.l.o.g., that $v_{1}$ is initially coloured red. Whenever Alice colours a vertex $v_{j}$ with $2 \leq j \leq n$, Bob colours $v_{j-1}$ if it is uncoloured. If $v_{j-1}$ is already coloured, then Bob colours the closest (in terms of its distance in the path) uncoloured vertex that is to the right of $v_{j}$. Towards a contradiction, assume that there exist 3 consecutive red vertices, denoted by $x_{1}, x_{2}, x_{3}$ from left to right in $P_{n}$. By Bob's strategy, concerning the 3 vertices $x_{1}, x_{2}, x_{3}$, Alice must have coloured $x_{1}$ first, then $x_{2}$, and then, $x_{3}$, as otherwise, Bob would have coloured at least one of them. But when Alice colours $x_{2}$, since $x_{1}$ is already coloured, then Bob will colour the closest uncoloured vertex to the right of $x_{2}$, which must be $x_{3}$ since it is uncoloured as it must get coloured by Alice after she colours $x_{2}$, and thus, we have a contradiction.

Lemma 2. Let $x \geq 1$ and $n \geq x$. Consider any path $P_{n}$ with $x$ vertices initially coloured blue, and let $y$ be the maximum order of an initial connected blue component.

- if $y=x$ and, either the blue component contains no ends of $P_{n}$ or $x=1$, then, if Alice starts, she has a strategy ensuring that Bob cannot create a connected blue component of order more than $x+1$;

- otherwise, if Alice starts, she has a strategy ensuring that Bob cannot create a connected blue component of order more than $x$.

Proof. First, let us consider the case $x=1$. If $n=1$, then the result is obvious. We prove the result by induction on $n$. Without loss of generality, let $v_{j}(1 \leq$ $j<n)$ be the vertex initially coloured blue. Then, Alice first colours $v_{j+1}$. Let $Q=\left(v_{1}, \ldots, v_{j}\right)$ and $Q^{\prime}=\left(v_{j+2}, \ldots, v_{n}\right)$ (it may be that $Q^{\prime}$ is empty and/or $Q$ is restricted to one vertex). From now on, Alice "follows" Bob, that is, when Bob plays in $Q$ (in $Q^{\prime}$, resp.), Alice then plays in $Q$ (in $Q^{\prime}$, resp.), and both games are considered independently (since $v_{j+1}$ is coloured red). Considering $Q$ as a path with one of its ends initially coloured blue, and applying Lemma 1 to it (but with Bob as the first player), Alice has a strategy ensuring that Bob cannot create a connected blue component of order more than 2 in $Q$. On the other hand, after the first move of Bob in $Q^{\prime}$, it is a path of order less than $n$ with one vertex initially coloured blue and it is the turn of Alice. Thus, by induction (on $n$ ), Alice has a strategy ensuring that Bob cannot create a connected blue 
component of order more than 2 in $Q^{\prime}$. Overall, Alice ensures that the largest connected blue component has order at most $2=x+1$. Hence, the claim holds for $x=1$.

Let $x>1$ and let us assume by induction that the previous statement holds for all $x^{\prime}<x$.

- Let us first assume that $y=x>1$ and the connected blue component $B$ contains no ends of $P_{n}$, say $B=\left(v_{i}, \ldots, v_{i+x-1}\right), 1<i<n-x+1$. Alice first colours $v_{i+x}$. If Bob colours $v_{i-1}$ on his next turn (in which case there is a connected blue component of order $x+1$ ), then Alice colours $z=v_{i-2}$ (unless $i=2$, in which case Alice colours an arbitrary uncoloured vertex). Otherwise, Alice colours $z=v_{i-1}$ (in which case the largest connected blue component is of order $x)$. Let $Q=\left(v_{1}, \ldots, z\right)$ and $Q^{\prime}=\left(v_{i+x+1}, \ldots, v_{n}\right)$ (it may be that $Q$ and/or $Q^{\prime}$ are empty, and, in particular, $Q$ is empty if $\left.z \notin\left\{v_{i-2}, v_{i-1}\right\}\right)$. From now on, Alice "follows" Bob, that is, when Bob plays in $Q$ (in $Q^{\prime}$, resp.), Alice then plays in $Q$ (in $Q^{\prime}$, resp.), and both games are considered independently (since $z$ and $v_{i+x}$ are coloured red). After the first move of Bob in $Q\left(Q^{\prime}\right.$, resp.), it is a path of order less than $n$ with at most $2 \leq x$ vertices initially coloured blue and it is the turn of Alice. Thus, by induction (on $n$ ), Alice has a strategy ensuring that Bob cannot create a connected blue component of order more than $x+1$ in $Q\left(Q^{\prime}\right.$, resp.). Overall, Alice ensures that the largest connected blue component in $P_{n}$ is of order at most $x+1$. Hence, the claim holds in this case.

- Next, let us assume that $y=x>1$ and the connected blue component $B$ contains one end of $P_{n}$, i.e., $B=\left(v_{1}, \ldots, v_{x}\right)$. Alice first colours $v_{x+1}$. Then, Bob colours any vertex in the subpath $Q=\left(v_{x+2}, \ldots, v_{n}\right)$. Therefore, $Q$ initially has one blue vertex and it is the turn of Alice. By the base case of the induction $(x=1)$, Alice can ensure that the largest connected blue component in $Q$ is of order at most 2. Overall, the largest connected blue component in $P_{n}$ is of order at most $x$. Hence, the claim holds in this case.

- Finally, let us assume that $y<x$. Let $\left(v_{i}, \ldots, v_{i+y-1}\right)$ be a largest connected blue component such that there is an initial blue vertex $v_{j}$ with $j>i+y$. Alice first colours $v_{i+y}$. Let $Q=\left(v_{1}, \ldots, v_{i+y-1}\right)$ and $Q^{\prime}=\left(v_{i+y+1}, \ldots, v_{n}\right)$ (it may be that $Q$ and/or $Q^{\prime}$ is empty). From now on, Alice "follows" Bob, that is, when Bob plays in $Q$ (in $Q^{\prime}$, resp.), Alice then plays in $Q$ (in $Q^{\prime}$, resp.), and both games are considered independently (since $v_{i+y}$ is coloured red). After the first move of Bob in $Q\left(Q^{\prime}\right.$, resp.), it is a path of order less than $n$ with at most $y+1 \leq x$ vertices initially coloured blue (and if there is a connected blue component with $x$ vertices, it must be in $Q$ and it contains the end $v_{i+y-1}$ of the path $Q$ ) and it is Alice's turn. Thus, by induction (on $n)$, Alice has a strategy ensuring that Bob cannot create a connected blue component of order more than $x$ in $Q\left(Q^{\prime}\right.$, resp.). Overall, Alice ensures that the largest connected blue component in $P_{n}$ is of order at most $x$. Hence, the claim holds in this case, and in general, since this is the last case.

Lemma 3. Let $x \geq 3, n \geq x+1$, and let $n-x$ be odd. Consider any path $P_{n}$ with $x$ vertices, including both ends, initially coloured blue. If Alice starts, then 
she has a strategy ensuring that Bob cannot create a connected blue component of order more than $x-1$ in $P_{n}$.

Proof. The first case, $x=3$, is proven by induction on $n$. If $n=4$, the result obviously holds, so assume that $n>4$ and that the induction holds for all $n^{\prime}<n$.

- First, assume that the initial blue vertices are $v_{1}, v_{2}$, and $v_{n}$. Then, Alice colours $v_{3}$. Then, Bob colours any uncoloured vertex in $Q=\left(v_{4}, \ldots, v_{n}\right)$. Now, $Q$ has 2 blue vertices (and if there is a connected blue component of order 2 in $Q$, it contains the end $v_{n}$ of $Q$ ). By Lemma 2, Alice can ensure that Bob cannot create a connected blue component with more than 2 vertices in $Q$. Overall, Bob cannot create a connected blue component of order at least 3 in $P_{n}$.

- Next, let $v_{1}, v_{j}, v_{n}$ (with $2<j<n-1$ ) be the initial blue vertices. W.l.o.g. (up to reversing the path), assume that $j$ is even (note that $n$ is even since $n-x=n-3$ is odd). Then, Alice colours $v_{j+1}$. Let $Q=\left(v_{1}, \ldots, v_{j}\right)$ and $Q^{\prime}=\left(v_{j+2}, \ldots, v_{n}\right)$ (it may be that $Q^{\prime}$ is just the vertex $\left.v_{n}\right)$. From now on, Alice "follows" Bob, that is, when Bob plays in $Q$ (in $Q^{\prime}$, resp.), Alice then plays in $Q$ (in $Q^{\prime}$, resp.), and both games are considered independently (since $v_{j+1}$ is coloured red). For the game in $Q^{\prime}$, applying Lemma 1 (but with Bob as the first player), Alice can ensure the largest connected blue component is of order at most 2 in $Q^{\prime}$. For the game in $Q$, by induction on $n^{\prime}=|Q|<n$ (note that, because $n^{\prime}=j$ is even, after the first turn of Bob in $Q$, the hypotheses hold for $x=3$ in $Q$ ), Alice can ensure the largest connected blue component is of order at most 2 in $Q$. Overall, Bob cannot create a connected blue component of order at least 3 in $P_{n}$.

Now, let us assume that $x>3$.

First, if there is a connected blue component of order $x-1$ containing $v_{1}$, then Alice colours $v_{x}$, and then she can ensure, by Lemma 1, that Bob cannot create a connected blue component with more than two vertices in $\left(v_{x+1}, \ldots, v_{n}\right)$.

Next, assume that there exists a blue component $\left(v_{j}, \ldots, v_{j+x-3}\right)$ of order $x-2$ not containing any end of $P_{n}$. W.l.o.g., let $j-2 \leq n-j-x+2$. Alice first colours $v_{j-1}$. Let $Q=\left(v_{1}, \ldots, v_{j-2}\right)$ and $Q^{\prime}=\left(v_{j}, \ldots, v_{n}\right)$. From now on, Alice "follows" Bob, that is, when Bob plays in $Q$ (in $Q^{\prime}$, resp.), Alice then plays in $Q$ (in $Q^{\prime}$, resp.), and both games are considered independently (since $v_{j-1}$ is coloured red). Note that, since $n-x$ is odd and $j-2 \leq n-j-x+2$, $Q^{\prime}=\left(v_{j}, \ldots, v_{n}\right)$ is of order at least $x+1$. When Bob plays in $Q$, Alice can ensure, by Lemma 1 , that Bob cannot create a connected blue component with more than two vertices in $Q$. When Bob first plays in $Q^{\prime}$, then $Q^{\prime}$ becomes a path of order at least $x+1$ with $x$ initial blue vertices, and its largest connected blue component contains its end $v_{j}$ and is of order at most $x-1$. By Lemma 2, Alice can ensure that Bob does not create a connected blue component of order more than $x-1$ in $Q^{\prime}$.

Otherwise, there must be an uncoloured vertex $v_{j}$ such that at most $x-2$ blue vertices are on the left (on the right, resp.) of $v_{j}$. Then, Alice first colours $v_{j}$. Let $Q=\left(v_{1}, \ldots, v_{j-1}\right)$ and $Q^{\prime}=\left(v_{j+1}, \ldots, v_{n}\right)$. From now on, Alice "follows" 


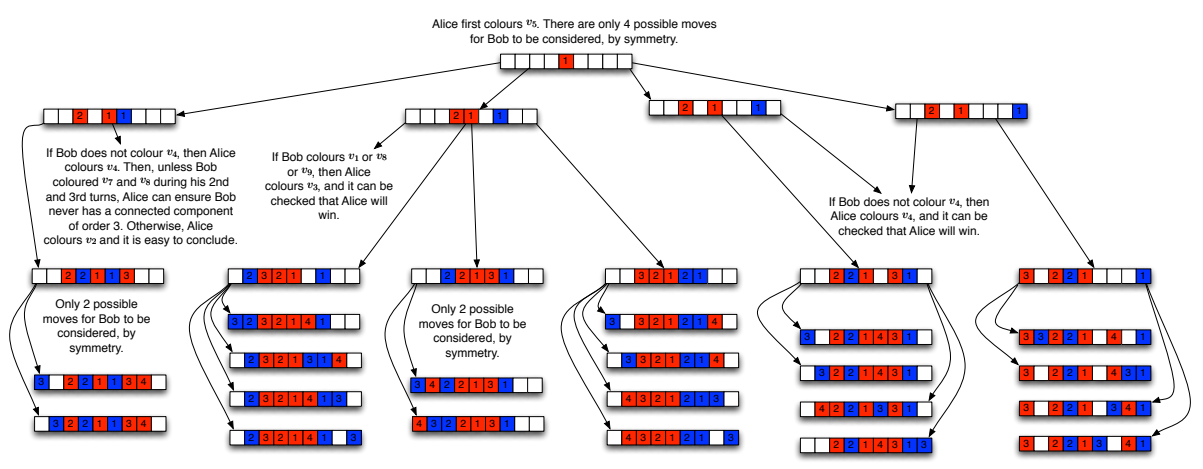

Fig. 2. Winning strategy for Alice in $P_{9}$. The vertices are represented by small squares. A number $i$ in a red (blue, resp.) square indicates that this vertex is the $i^{\text {th }}$ vertex coloured by Alice (Bob, resp.). Every arrow corresponds to two moves: first a move of Bob and then of Alice. The leaves of the game-tree correspond to paths where there are still two vertices uncoloured. It is then easy to check the last possibilities.

Bob, that is, when Bob plays in $Q$ (in $Q^{\prime}$, resp.), Alice then plays in $Q$ (in $Q^{\prime}$, resp.), and both games are considered independently (since $v_{j}$ is coloured red). By Lemma 2, Alice can ensure, both in $Q$ and $Q^{\prime}$, that Bob does not create a blue component with at least $x$ vertices (note that after the first turn of Bob in $Q\left(Q^{\prime}\right.$, resp $)$ it contains at most $x-1$ blue vertices including at least one of its ends).

Theorem 5. For all $n \geq 1$, the path $P_{n}$ is $A$-win if and only if $n \in\{1,3,5,7,9\}$.

Missing part of the proof. [Small values of $n$ ] We need to show that, if $n \in$ $\{1,3,5,7,9\}$, then $P_{n}$ is $A$-win. It is easy to see that, if $n \leq 7$ and $n$ is odd, then Alice wins by first colouring the center of $P_{n}$. If $n=9$, a winning strategy for Alice is described in Figure 2.

Theorem 7. Let $G$ be a cograph. There exists a linear-time algorithm that decides whether $G$ is $A$-win or $A B$-draw, and whether $G \in \mathcal{A}^{*}$ or not.

Missing part of the proof. [Case 1] Let us assume that $\boldsymbol{G}=\boldsymbol{G}_{\mathbf{1}} \oplus \boldsymbol{G}_{\mathbf{2}}$. There are three cases to be distinguished.

1. If $n$ is odd (so we may assume that $\left|V\left(G_{2}\right)\right| \geq 2$ ), then $G$ is $A$-win and $G \in \mathcal{A}^{*}$.

Alice first colours a vertex in $G_{1}$. In the second round, Alice colours a vertex in $G_{2}$ (it is possible since $\left|V\left(G_{2}\right)\right| \geq 2$ ). Then, Alice colours any uncoloured vertex in each of the remaining rounds. Regardless of Bob's strategy, Alice ends with all the $\left\lceil\frac{n}{2}\right\rceil$ red vertices belonging to the same connected component. Since $n$ is odd, $G$ is $A$-win and $G \in \mathcal{A}^{*}$. 
2. If $\left|V\left(G_{1}\right)\right|,\left|V\left(G_{2}\right)\right| \geq 2$ and $n$ is even, then $G$ is $A B$-draw and $G \in \mathcal{A}^{*}$. W.l.o.g., Alice first colours a vertex in $G_{1}$. Then, Bob first colours a vertex in $G_{1}$ (it is possible since $\left|V\left(G_{1}\right)\right| \geq 2$ ). In the second round, Bob colours a vertex in $G_{2}$ (it is possible since $\left|V\left(G_{2}\right)\right| \geq 2$ ). Then, Bob colours any uncoloured vertex in each of the remaining rounds. Regardless of Alice's strategy, Bob ends with all the $n / 2$ blue vertices belonging to the same connected component. Since $n$ is even, Alice cannot have a larger connected red component. Hence, $G$ is $A B$-draw and $G \in \mathcal{A}^{*}$.

3. Finally, let us assume that $\left|V\left(G_{1}\right)\right|=1$ (let $u$ be the single vertex of $G_{1}$ ) and $n$ is even (so $\left|V\left(G_{2}\right)\right|$ is odd). There are two cases to be considered.

(a) If $G_{2} \notin \mathcal{A}^{*}$, then $G$ is $A$-win and $G \in \mathcal{A}^{*}$.

Indeed, Alice first colours $u$. Then, she plays in $G_{2}$ as the second player, and thus, she can ensure that any connected blue component is of order less than $\left\lceil\frac{\left|V\left(G_{2}\right)\right|}{2}\right\rceil=\left\lceil\frac{n-1}{2}\right\rceil$ in $G_{2}$ since $G_{2} \notin \mathcal{A}^{*}$. Since $u$ is a universal vertex, regardless of Bob's strategy, Alice ensures a connected red component of order $n / 2$, and so $G$ is $A$-win and $G \in \mathcal{A}^{*}$.

(b) If $G_{2} \in \mathcal{A}^{*}$, then $G$ is $A B$-draw and $G \in \mathcal{A}^{*}$.

If Alice first colours a vertex of $G_{2}$, then Bob colours $u$, and then Bob colours any uncoloured vertex of $G_{2}$ in each of the subsequent rounds. Then, Bob ensures a connected blue component of order $n / 2$, and so $G$ is $A B$-draw and $G \in \mathcal{A}^{*}$.

Otherwise, if Alice starts by colouring $u$, then Bob can play as the first player in $G_{2}$ and, in doing so, ensure a connected blue component of order $\left\lceil\frac{n-1}{2}\right\rceil=n / 2$ in $G_{2}$. Then, again $G$ is $A B$-draw and $G \in \mathcal{A}^{*}$. 